\title{
How I do it: The mccabe modification for endoscopic removal of an endoluminal airway stent
}

\author{
Weinstock MS ${ }^{1,2}$ and Smith LP' \\ ${ }^{1}$ Cohen Children's Medical Center, Hofstra Northwell School of Medicine, New Hyde Park, NY \\ ${ }^{2}$ Montefiore Medical Center, Albert Einstein College of Medicine, Bronx NY
}

\begin{abstract}
The safe and efficient removal of endoluminal airway stents is an essential process in airway reconstruction surgery. Through trial and error, we have devised a novel approach to accomplishing this task. The following article chronicles this method and provides a step-wise approach to aid the surgeon in the usage of this technique as he or she deems fit.
\end{abstract}

\section{Introduction}

Open airway reconstruction can be performed in a single stage LTR (ssLTR) or double stage (dsLTR) fashion. When surgery is performed, the newly reconstructed airway is often stented while healing from the procedure. In single stage procedures, this stent is typically an endotracheal tube [1]. For double stage procedures, an endoluminal stent is often left in place. This stent is secured in place with a suture. In this report, we provide an efficient method for removing this stent using both percutaneous and intraluminal methods.

\section{Technique}

The senior surgeon will usually use a cylindrical silastic stent for dsLTR. The endoluminal stent is usually placed at the area of reconstruction with the superior limb at the level of the false vocal cords and the inferior limb at the level of the tracheostomy (Figure

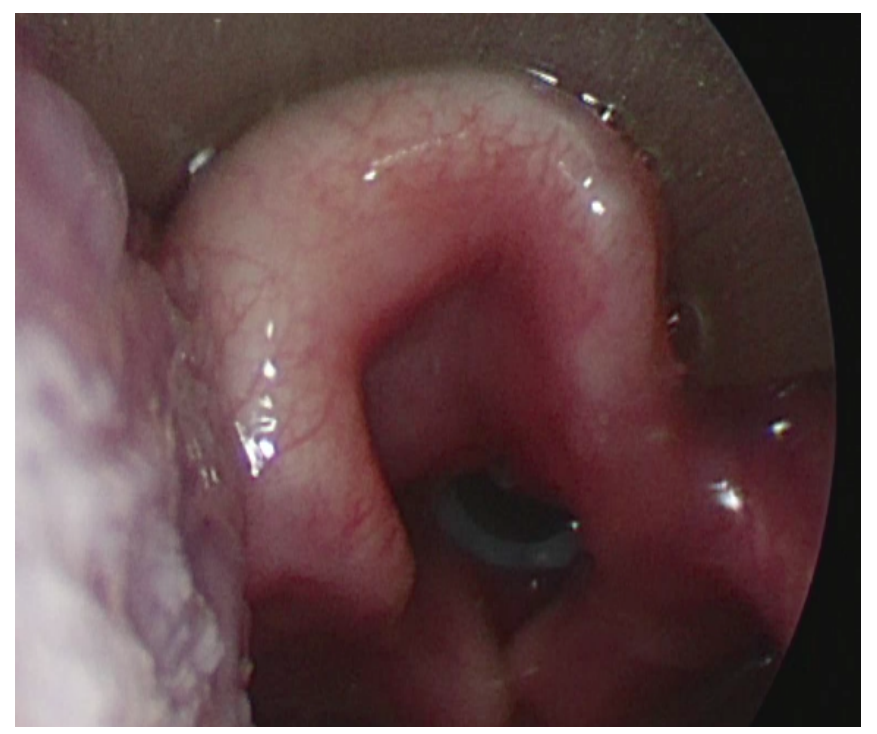

Figure 1. Endoluminal stent placed at level of the glottis, extending to the subglottis, covering the area of reconstruction
1). The stent is secured in place using a 2-0 prolene suture. The stent suture is passed from the transervical incision and passed through the upper trachea and through the endolumen of the stent to secure the stent in place. The suture is then tied with roughly 20 knots that emanate from the right side of the transcervical incision (Figure 2). Usually seven days after surgery, the child is brought back to the OR for stent removal.

During this procedure, we perform direct laryngoscopy and using a 0-degree Hopkins rod telescope (Karl Storz, USA, El Segundo, CA) we visualize the endolumen of the stent and under bronchoscopic visualization, we cut the stent stitch with a laryngeal scissors (Figure 3). We then secure the stent using an endoscopic alligator clamp. The

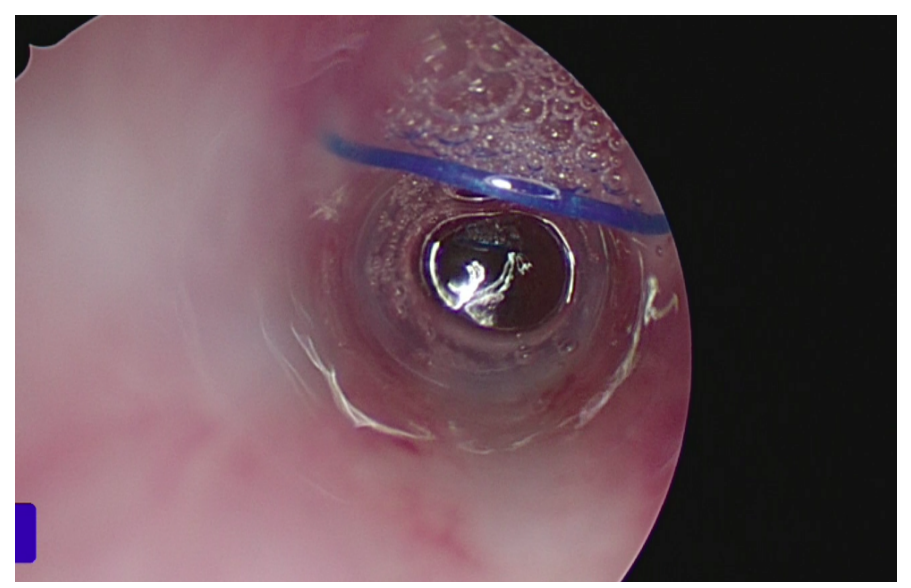

Figure 2. Endoluminal Stent with 2-0 Prolene suture in place

${ }^{*}$ Correspondence to: Smith LP, MD, Steven and Alexandra Cohen Children's Medical Center, Hofstra Northwell School of Medicine, 430 Lakeville Rd. New Hyde Park, NY 11040. E-mail: Lsmith8@northwell.edu

Key words: Double Stage LTR (dsLTR), Endoluminal Stent

Received: May 05, 2018; Accepted: May 22, 2018; Published: May 25, 2018 


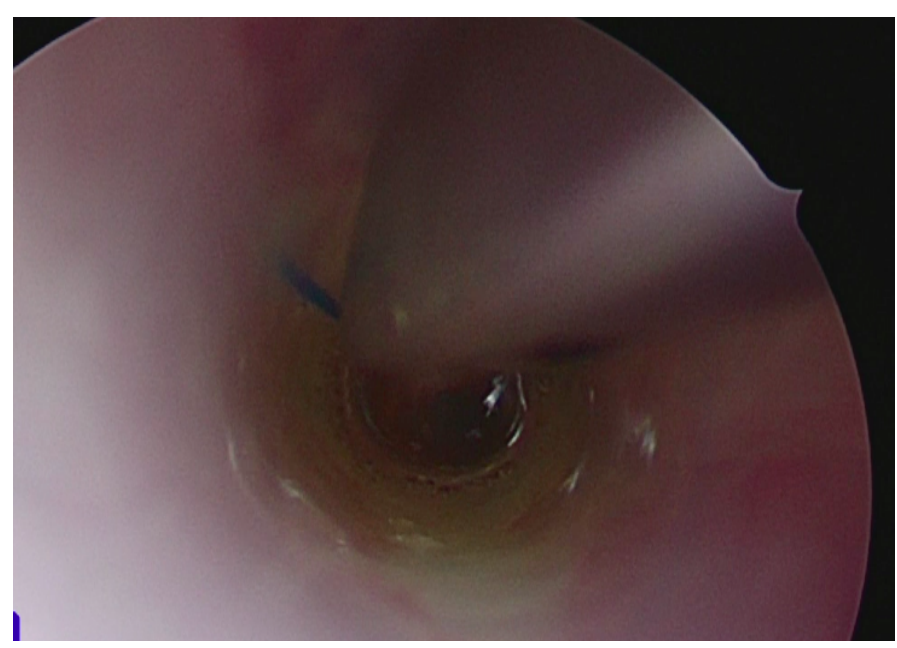

Figure 3. Cutting the endoluminal suture with endoscopic scissor.

portion of the stitch emanating from the right side of the skin incision is grasped and removed percutaneously. The stent then can be removed transorally with the alligator clamp.
Discussion: Previously, our technique included the percutaneous cutting and removal of the prolene suture rather than the intraluminal cutting and percutaneous removal of the prolene suture. We have found that by cutting the suture intraluminally we save several precious operating room minutes. We also are able to directly visualize the entirety of the procedure within the airway, making the removal of the stent and suture a safer procedure as the we have complete control over the stent throughout the procedure.

\section{Conflicts of Interest}

None

\section{Acknowledgements}

None

\section{Funding sources}

No funding sources

\section{Reference}

1. Prescott CA (1988) Protocol for management of the interposition cartilage graft laryngotracheoplasty. Ann Otol Rhinol Laryngol 97: 239- 242. [Crossref]

Copyright: $@ 2018$ Weinstock MS. This is an open-access article distributed under the terms of the Creative Commons Attribution License, which permits unrestricted use, distribution, and reproduction in any medium, provided the original author and source are credited. 